\title{
Association of Chronic Pain with Radiologic Severity and Central Sensitization in Hip Osteoarthritis Patients
}

\author{
Takafumi Hattori ${ }^{1,2}$ \\ Kazuhiro Shimo ${ }^{3}$ \\ Yuto Niwa' \\ Yuji Tokiwa' \\ Takako Matsubara ${ }^{1,3}$ \\ 'Faculty of Rehabilitation, Kobe Gakuin \\ University Graduate School, Kobe, \\ Hyogo, Japan; ${ }^{2}$ Department of \\ Rehabilitation, Maehara Orthopedics \\ Rehabilitation Clinic, Obu, Aichi, Japan; \\ ${ }^{3}$ Department of Physical Therapy, Faculty \\ of Rehabilitation, Kobe Gakuin University, \\ Kobe, Hyogo, Japan
}

Purpose: Pain and joint deformity are the most common symptoms of hip osteoarthritis (OA). However, no significant association between pain and severity of radiographic lesions has been reported. Recently, central sensitization has been suggested as an underlying mechanism of pain in OA. We investigated the involvement of radiologic severity or central sensitization in the clinical manifestation of hip OA with various degrees of joint deformity.

Patients and Methods: We included 39 patients with hip OA and divided them into two groups according to the severity of the hip pain: strong/severe (numerical rating scale, $\mathrm{NRS} \geq 6$ ) and mild/moderate (NRS $<6$ ). We assessed the radiologic severity of OA using the Kellgren-Lawrence (K-L) scale and minimum joint space width (mJSW). We conducted quantitative sensory testing (QST) that included pressure pain threshold (PPT) and temporal summation of pain (TSP) at hip, tibialis anterior (leg), and extensor carpi radialis longus (arm) on the affected side. We examined the difference of radiologic assessment and QST results between each group and the correlation of the NRS with the radiologic assessment and QST results.

Results: There was no significant difference in the K-L scale and mJSW between patients with strong/severe and mild/moderate joint pain. Strong/severe pain patients demonstrated a lower PPT at all measurement sites and higher TSP at the hip and leg than the mild/moderate pain patients. In addition, NRS was significantly negatively correlated with PPT and positively correlated with TSP at all measurement sites, but not with the K-L scale and mJSW.

Conclusion: We reported no significant difference in radiologic severity between patients with strong/severe and mild/moderate joint pain. By contrast, we found a significant difference in central sensitization represented by QST between strong/severe and mild/moderate joint pain groups. These results suggest that central sensitization may be involved in the joint pain of patients with hip OA who complain of severe pain despite less severe joint deformity.

Keywords: hip osteoarthritis, quantitative sensory testing, pressure pain threshold, temporal summation of pain

\section{Introduction}

Osteoarthritis (OA) is a chronic pain disorder based on nociceptive pain with joint structural damage. Hip $\mathrm{OA}$ is the most common musculoskeletal joint disease worldwide. ${ }^{1,2}$ Due to a growing elderly population, OA prevalence will continuously increase and present as a growing burden on the healthcare infrastructure and economy. ${ }^{3}$ In Japan, the prevalence of radiologic hip OA was $18.2 \%$ in men and $14.3 \%$ in women. ${ }^{4}$ Hip OA is characterized initially by cartilage degradation, which
Correspondence: Kazuhiro Shimo Department of Physical Therapy, Faculty of Rehabilitation, Kobe Gakuin University, 518 Arise, Ikawadani-cho, Nishi-ku, Kobe, Hyogo, 65I-2I80, Japan

Tel/Fax +8I-78-974-246I

Email shimo@reha.kobegakuin.ac.jp 
often precedes changes in the underlying bone. The prevalence of joint pain tends to increase with a deterioration of radiologic severity. ${ }^{4}$ However, the prevalence of hip OA patients with pain and Kellgren-Lawrence (K-L) scale $\geq 2$ was $0.29 \%$ in men and $0.99 \%$ in women. ${ }^{4}$ Moreover, it is estimated that up to $40 \%$ of individuals with radiologic evidence of damage have no pain. ${ }^{5}$ Moreover, a previous study on hip OA suggested no significant associations between the severity of radiographic lesions and pain or disability or quality of life. ${ }^{6}$ Furthermore, we often find a discrepancy between the physical damage to the joint and the level of self-reported pain in clinical practice. Therefore, there is no clear relationship between the findings of radiologic severity and pain symptoms reported by the patients.

In the recent years, peripheral and central sensitization has been proposed to contribute to pain in OA. The nociceptive inputs cause peripheral sensitization, which is a reduction in the activation threshold and amplification in the responsiveness of the primary afferent nociceptors. ${ }^{7}$ Although inflammation and joint damage may be the initial trigger for OA pain, the repeated input of noxious stimuli can induce neuronal plasticity and subsequent central sensitization. ${ }^{8}$ Central sensitization indicates a state where the central nervous system neurons become hyperexcitable to noxious and innocuous stimuli and involve multiple somatosensory processing changes. ${ }^{9}$ Previous studies have reported that central sensitization is a feature of chronic pain disorders, such as knee and hip OA, chronic low back pain, and fibromyalgia. ${ }^{10-13}$ In clinical practice, total hip arthroplasty (THA) is usually an effective surgical intervention to improve joint structural damage and provide pain relief in OA. However, $10 \%$ of patients had THA experience chronic postsurgical pain, ${ }^{14}$ and preoperative central sensitization has been reported to be a risk factor of chronic postsurgical pain. ${ }^{11}$

Quantitative sensory testing (QST) has also been used recently as one of the assessments that can evaluate mechanism-based phenotyping of OA pain. ${ }^{15}$ QST is known as psychophysical testing and refers to tests of sensory perception during the stimuli with predetermined physical properties and following specific protocols. ${ }^{15}$ QST measurements consist of static and dynamic QSTs. Static QST refers to the assessment of the states of the peripheral nervous system. ${ }^{16}$ Dynamic QST refers to the assessment of the pain mechanism of central processing beyond the peripheral nervous system. ${ }^{17}$ Pressure pain threshold (PPT) classified as a static QST is useful in assessing pain reactions in OA patients with or without sensitization. The QST study of knee OA showed that $34 \%$ of patients presented with pressure hyperalgesia at the knee. ${ }^{18}$ Temporal summation of pain (TSP) classified as a dynamic QST refers to the increased perception of pain in response to repetitive noxious stimuli delivered at frequencies above $0.3 \mathrm{~Hz}$. This is considered the hallmark of central sensitization. ${ }^{19}$ In patients with knee OA, prominent facilitated TSP features are linked to more pain symptoms. ${ }^{10}$ However, to our knowledge, few studies have investigated response to both static and dynamic QST in hip OA. Furthermore, previous studies targeted patients with severe joint deformity only. The influence of radiologic severity or QST results on joint pain in hip OA patients with various degrees of joint deformity is not yet clear.

The aim of this study was to determine whether radiologic severity of the joint deformity or central sensitization was more involved in pain of hip OA with K-L scale $1-4$. We hypothesized that self-reported hip joint pain and radiologic severity are not related, but central sensitization assessed using QST affects joint pain of hip OA.

\section{Patients and Methods}

\section{Subjects}

Thirty-nine patients with hip OA were included in the study. The study participants were selected from an orthopedic clinic in Obu, Japan. Inclusion criteria for the participants were a diagnosis of OA confirmed by radiographic findings; clinical hip pain as the primary musculoskeletal complaint at the time of recruitment; age of 40 years or above; and chronic hip pain for at least six months. Exclusion criteria were systemic inflammatory diseases; cognitive impairment affecting the ability to cooperate with testing; leg pain referred from the lumbar spine; and presence of serious medical comorbidities (eg congestive heart failure, cerebrovascular disease, and cancer). We divided the patients into two groups based on the peak self-reported pain intensity over the last $24 \mathrm{~h} .{ }^{10,20,21}$ The patients who had strong/severe joint pain (peak pain) over the last 24 $\mathrm{h}$ (numerical rating scale $[\mathrm{NRS}] \geq 6$ ) were assigned to group A and the patients who had mild/moderate peak pain over the last $24 \mathrm{~h}(\mathrm{NRS}<6)$ were assigned to group B. Therefore, central sensitization was assessed using systemic QSTs, such as PPT and TSP, and the difference of radiologic severity or QST results was 
investigated in groups of hip OA patients whose selfreported pain differed.

\section{Demographic and Clinical Data}

All participants were interviewed to obtain data on their characteristics (age, sex, body mass index [BMI], duration of pain symptoms, clinical pain intensity assessed on the NRS, disability, and neuropathic pain-like symptoms). Disability was assessed using the Function in Activities of Daily Living subscale of the Hip Disability and Osteoarthritis Outcome Score (HOOS). ${ }^{22,23}$ The questionnaire was valid for hip OA, with scores ranging from 0 (worst) to 100 (best) for each subscale. The painDETECT questionnaire was used to identify patients with potential neuropathic pain components. ${ }^{24} \mathrm{~A}$ recent study has suggested that pain sensitivity is associated with neuropathic pain-like symptoms in hip OA. ${ }^{25}$

\section{Radiographic Assessment of OA}

In all patients, posteroanterior radiographs of the hip with weight bearing were obtained. To assess the radiologic severity of OA, we used the K-L scale and minimum joint space width (mJSW). The characteristics for each K-L scale can be summarized as follows: grade 1, doubtful OA, with the presence of minor osteophytes of doubtful importance; grade 2, minimal OA, with definite osteophytes but an unimpaired joint space; grade 3, moderate $\mathrm{OA}$, with osteophytes and a moderate diminution of the joint space; and grade 4, severe OA, with a greatly impaired joint space and sclerosis of the subchondral bone. ${ }^{26}$ The mJSW has become the standard tool for the assessment of OA progression. ${ }^{27}$ The mJSW of the hip on the coronal radiograph refers to the interbone area between the acetabular roof and the part of the femoral head facing it. K-L scale and mJSW were evaluated by one orthopedic surgeon.

\section{Quantitative Sensory Testing}

QST consisted of PPT and TSP at the hip, the tibialis anterior (leg, $5 \mathrm{~cm}$ distal to the tibial tuberosity) and extensor carpi radialis longus ( $\mathrm{arm}, 5 \mathrm{~cm}$ distal to the lateral epicondyle of the humerus). The measurement order was PPT at the hip, leg, and arm, followed by TSP at the hip, leg, and arm in five-minute intervals. Patients laid down on a bed in either a supine or a lateral decubitus position for the measurement, and all measurements were taken by one physical therapist.

\section{Pressure Pain Threshold}

Four test sites in the hip, one control site on the leg, and one control site on the arm were located and marked. The four sites of the hip were located according to bony landmarks as follows: Hip-1: $3 \mathrm{~cm}$ proximal to the tip of the greater trochanter; Hip-2: $3 \mathrm{~cm}$ posterior to the posterior edge of the greater trochanter; Hip-3: $3 \mathrm{~cm}$ distal to the distal edge of the greater trochanter; Hip-4: $3 \mathrm{~cm}$ anterior to the anterior edge of the greater trochanter. ${ }^{11}$ The PPT value of the hip was defined as the site's value with the lowest PPT.

A hand-held pressure algometer (Algometer Type II, Somedic AB, Sweden) with a $1-\mathrm{cm}^{2}$ contact probe was used for measuring PPT. PPT was conducted on each site by continually applying pressure $(30 \mathrm{kPa} / \mathrm{s})$ until the patient defined the pressure as pain. PPT was measured twice at each site and the mean of the two measurements was used in the statistical analysis. The lower the PPT, the greater the degree of sensitization or pain sensitivity.

\section{Temporal Summation of Pain}

TSP, an augmented response to repetitive mechanical stimulation, is a sensitive and valid measure of the central pain processing, which is a feature of central sensitization. A hand-held pressure algometer was used to apply mechanical stimuli perpendicular to the skin surface. The stimulation consisted of ten pressure stimuli (1-s duration and 1-s interval) at the PPT level. ${ }^{10,28}$ The stimulation was applied once to the hip joint (the most sensitive part out of the four sites where PPT was measured) and to the control site on the leg and arm. Skin contact between the individual pressure stimuli was ensured by maintaining a constant force. The contact force between the two stimuli did not induce pain. The subjects rated the pain intensity continuously during the sequential stimulation on a visual analogue scale (VAS) where " 0 " indicated "no pain," and "100" indicated "worst possible pain." TSP was normalized by subtraction from the VAS scores to the first stimulus, and the 10th VAS defined the value of TSP. ${ }^{29}$

\section{Statistical Analysis}

Data are presented as mean and standard deviation (SD). As most of our data were not normally distributed, we used the less sensitive but more robust nonparametric tests in all statistical analyses. Demographic and clinical data, radiologic severity, and QST results were compared between group A and B, respectively, using the Mann-Whitney $U$-test and 
Fisher's exact test. We also used " $\mathrm{r}$ " as calculated by $\mathrm{Z}$ translation to evaluate the magnitude of the effect size $(\mathrm{r}=\mathrm{Z} / \sqrt{ } \mathrm{N})$ according to Cohen. ${ }^{30}$ The correlations between NRS and K-L scale, mJSW, PPT, or TSP were evaluated, and the Spearman's rank test was used for correlation analysis. A significance level of $\mathrm{p}<0.05$ was used for each analysis.

\section{Results}

\section{Demographic and Clinical Data}

Fifteen patients were categorized into the strong/severe joint pain group (group A, NRS: 7.0 \pm 0.9 ) and 24 were categorized into the mild/moderate joint pain group (group B, NRS: $3.0 \pm 1.1$ ). The demographic and clinical data of the patients are shown in Table 1. No significant differences between the groups were found in age $(60.1 \pm 14.2$ for group A; $57.4 \pm 11.0$ for group $\mathrm{B}, \mathrm{p}=0.69$ ), sex (male: 1 [6\%], female: 14 [94\%] for group A; male: 3 [12\%], female: 21 [88\%] for group B, $\mathrm{p}=1.00)$, and $\mathrm{BMI}(23.9 \pm 2.9$ for group $\mathrm{A} ; 23.5 \pm 3.1$ for group $B, p=0.77)$. There were significant differences between groups $\mathrm{A}$ and $\mathrm{B}$ in pain duration $(64.8 \pm 49.5$ months for group $A ; 38.2 \pm 60.2$ months for group $B, r=0.35, p<0.05)$, HOOS (54.5 \pm 21.3 for group A; $82.5 \pm 13.8$ for group $B$, $\mathrm{r}=0.62, \mathrm{p}<0.001)$, and painDETECT $(12.4 \pm 4.4$ for group $\mathrm{A} ; 4.5 \pm 3.5$ for group $\mathrm{B}, \mathrm{r}=0.67, \mathrm{p}<0.001)$. In painDETECT, 8 patients in group A were classified as nociceptive (total score of painDETECT $<13)$, 5 were unclear $(13 \leq$ score $<19)$, and 2 were neuropathic $(\geq 19)$. All patients in group B were classified as nociceptive (total score of painDETECT $<13$ ). The analgesics used by the patients up to $24 \mathrm{~h}$ before the examination were divided into NSAIDs, acetaminophen,

Table I Demographic and Clinical Data of Each Group

\begin{tabular}{|l|l|l|l|}
\hline Variables & Group A & Group B & P value \\
\hline Number & 15 & 24 & \\
\hline Age (years) & $60.1 \pm 14.2$ & $57.4 \pm 11.0$ & 0.69 \\
\hline $\begin{array}{l}\text { Sex, number (\%) } \\
\text { Male } \\
\text { Female }\end{array}$ & $\begin{array}{l}1(6) \\
14(94)\end{array}$ & $\begin{array}{l}3(12) \\
21(88)\end{array}$ & 1.00 \\
\hline BMI & $23.9 \pm 2.9$ & $23.5 \pm 3.1$ & 0.77 \\
\hline Pain duration (months) & $64.8 \pm 49.5$ & $38.2 \pm 60.2$ & $<0.05$ \\
\hline Clinical pain intensity (NRS) & $7.0 \pm 0.9$ & $3.0 \pm 1.1$ & $<0.001$ \\
\hline HOOS (function in ADL) & $54.5 \pm 21.3$ & $82.5 \pm 13.8$ & $<0.001$ \\
\hline painDETECT & $12.4 \pm 4.4$ & $4.5 \pm 3.5$ & $<0.001$ \\
\hline
\end{tabular}

Abbreviations: BMI, body mass index; NRS, numerical rating scale; HOOS, Hip Disability and Osteoarthritis Outcome Score; ADL, activities of daily living. duloxetine, pregabalin, and opioids. The number of patients using these medications was 10, 0, 2, 0, and 2 in group A and $5,1,0,0$, and 0 in group $\mathrm{B}$, respectively.

\section{Radiographic Assessment of OA}

$\mathrm{K}-\mathrm{L}$ scale from both groups is shown Table 2 . There were $5,4,2$, and 4 patients in group $A$ and 8, 7, 5, and 4 patients in group B classified into K-L scale 1, 2, 3, and 4, respectively. There was no difference in the percentage of subjects included in each grade $(p=0.88)$. No difference was found between group A and B for mJSW (2.6 \pm 1.9 for group $\mathrm{A} ; 2.7 \pm 1.3$ for group $\mathrm{B}, \mathrm{p}=0.96$ ) (Figure 1).

\section{Pressure Pain Threshold}

The PPT from both groups is shown in Figure 2. The PPT at the hip in Group A was lower than that in Group B (186.3 \pm 82.0 for group A; $322.0 \pm 102.9$ for group B,

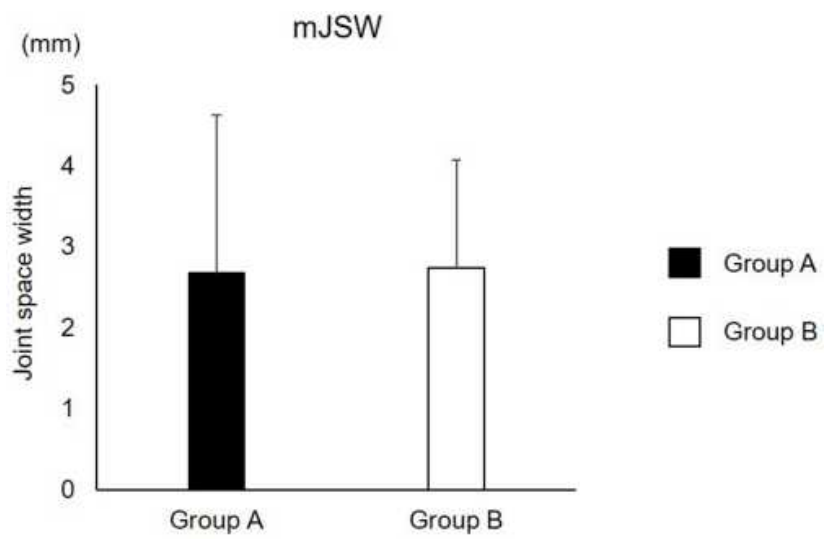

Figure I mJSW.

Abbreviation: $\mathrm{mJSW}$, minimum joint space width.

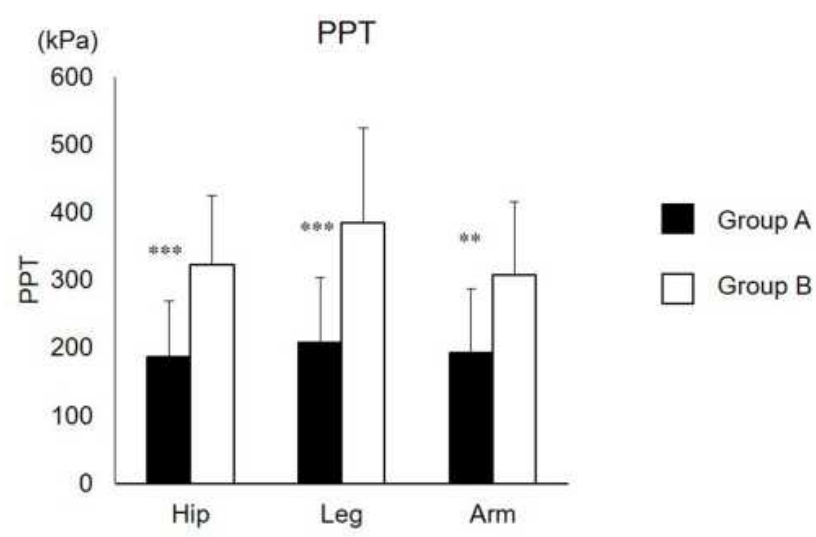

Figure 2 PPT at the hip, tibialis anterior, and extensor carpi radialis longus. Note: **, ***Significantly lower than group $B(p<0.01,0.001)$.

Abbreviations: PPT, pressure pain threshold; Leg, tibialis anterior; Arm, extensor carpi radialis longus. 


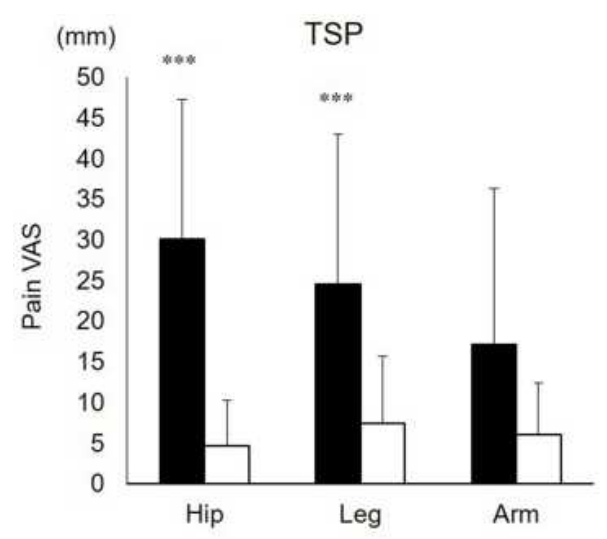

Figure 3 TSP at hip, tibialis anterior, and extensor carpi radialis longus. Note: ***Significantly higher than group $B(p<0.00 I)$.

Abbreviations: TSP, temporal summation of pain; VAS, visual analogue scale; Leg, tibialis anterior; Arm, extensor carpi radialis longus.

$\mathrm{r}=0.60, \mathrm{p}<0.001)$. PPT at leg in group A was lower than that in Group B $(208.2 \pm 96.1$ for group A; $384.0 \pm 139.9$ for group $\mathrm{B}, \mathrm{r}=0.58, \mathrm{p}<0.001)$. PPT at arm in Group A was lower than that in group B $(192.6 \pm 94.9$ for group A; 307.8 \pm 107.8 for group $\mathrm{B}, \mathrm{r}=0.52, \mathrm{p}<0.01)$.

\section{Temporal Summation of Pressure Pain}

The TSP from both groups is shown in Figure 3. TSP at the hip in group A was significantly higher than that in group B (30.1 \pm 17.2 for group A; 4.7 \pm 5.6 for group B, $\mathrm{r}=0.79, \mathrm{p}<0.001)$. TSP at leg in group A was significantly higher than that in group $\mathrm{B}(24.5 \pm 18.5$ for group $\mathrm{A} ; 7.3$ \pm 8.3 for group $B, r=0.53, p<0.001)$. No significant difference was found between groups $\mathrm{A}$ and $\mathrm{B}$ for TSP at arm (17.0 \pm 19.2 for group $A ; 6.0 \pm 6.3$ for group $B, p=0.051)$.

\section{Correlations}

The relationship between NRS and radiographic assessment or QST results was analyzed and is shown in Table 3. There was no correlation between NRS and K-L scale or NRS $(p=0.18)$ and mJSW ( $p=0.21)$. Individual NRS was significantly correlated with hip PPT ( $\mathrm{rs}=-0.511, \mathrm{p}<0.01)$,

Table 2 K-L Scale

\begin{tabular}{|l|l|l|c|}
\hline Variable & Group A & Group B & P value \\
\hline \multicolumn{4}{|l|}{ K-L scale, number (\%) } \\
\hline 1 & $5(33)$ & $8(33)$ & 0.88 \\
2 & $4(27)$ & $7(29)$ & \\
3 & $2(13)$ & $5(21)$ & \\
4 & $4(27)$ & $4(17)$ & \\
\hline
\end{tabular}

Abbreviation: K-L, Kellgren-Lawrence.
Table 3 Correlation Between NRS and Radiologic Severity or QST Results

\begin{tabular}{|l|l|l|}
\hline Variables & rs & P value \\
\hline K-L scale & 0.218 & 0.18 \\
\hline mJSW & -0.202 & 0.21 \\
\hline PPT & & \\
Hip & -0.511 & $<0.01$ \\
Leg & -0.572 & $<0.01$ \\
Arm & -0.548 & $<0.01$ \\
\hline TSP & & \\
Hip & 0.717 & $<0.01$ \\
Leg & 0.592 & $<0.01$ \\
Arm & 0.430 & $<0.01$ \\
\hline
\end{tabular}

Abbreviations: NRS, numerical rating scale; K-L, Kellgren-Lawrence; mJSW, minimum joint space width; PPT, pressure pain threshold; TSP, temporal summation of pain; Leg, tibialis anterior; Arm, extensor carpi radialis longus.

leg PPT ( $\mathrm{rs}=-0.572, \mathrm{p}<0.01)$, arm PPT $\quad(\mathrm{rs}=-0.548$, $\mathrm{p}<0.01$ ), hip TSP ( $\mathrm{rs}=0.717, \mathrm{p}<0.01)$, leg TSP ( $\mathrm{rs}=0.592$, $\mathrm{p}<0.01$ ) (Table 3), and arm TSP (rs=0.430, $<<0.01)$.

\section{Discussion}

This study investigated whether radiologic severity or central sensitization was more involved in the clinical manifestation of hip OA. In the present study, patients in the strong/severe pain group had a longer pain duration and more functional disability and neuropathic pain-like symptoms than patients with mild/moderate joint pain. Furthermore, we found a significant difference in QST results between patients with strong/severe and mild/ moderate joint pain. Patients with strong/severe pain showed a lower PPT and higher TSP than those with mild/moderate pain. In addition, QST was significantly correlated with joint pain. By contrast, our results showed no significant differences in the K-L scale and mJSW between patients with strong/severe and mild/moderate joint pain. Our results suggest that not only joint deformity but also central sensitization influences the hip OA pain.

Pain and joint deformity are the most common symptoms of hip OA. ${ }^{1,2}$ The prevalence of joint pain tends to increase with the deterioration of radiologic severity. ${ }^{4}$ Thus, the pain of hip OA is considered nociceptive pain associated with joint deformity. ${ }^{31}$ However, in clinical practice, many patients report a high level of pain without a severe radiologic deformity and vice versa. A previous cross-sectional study showed no significant associations 
between the severity of radiographic deformity and joint pain in patients with hip OA. ${ }^{6}$ As expected, our results were in alignment with those of the previous report. These observations indicate that joint deformity has a limited effect on pain in hip OA.

In our study, patients with strong/severe pain showed a lower PPT and a higher TSP than did those with mild/ moderate pain in the affected hip joint and non-affected sites, such as the lower leg and forearm, while there were no significant differences in the K-L scale and mJSW between groups. In particular, TSP is also a sensitive measure of central sensitization, ${ }^{32}$ and facilitated TSP can be found in many painful chronic musculoskeletal conditions such as chronic low back pain and fibromyalgia. $^{12,13}$ Our results demonstrate that patients with painful hip OA have a more facilitated central sensitization than those with mild pain. Repeated pain input to the affected joint in OA may be accompanied by increased tenderness of the lower leg and forearm muscles and may cause central spreading sensitization. Studies on animals have reported that noxious stimuli to a specific receptive field in a muscle generated new muscle receptive fields at a distance from the original one within minutes. ${ }^{33,34}$ The spread of sensitization to adjacent spinal segments has been proposed to account for the observed phenomenon and the spread of pain to larger body areas. In a previous knee OA study, patients with strong/severe pain showed widespread hyperalgesia compared to those with mild/ moderate pain. ${ }^{10}$ Our hip OA findings are similar to the findings of previous knee OA studies. For correlations, we found that QST results were significantly correlated with joint pain in hip OA. These results show that indicators of central sensitization extend beyond the affected joint to the affected leg and forearm in patients with hip OA with varying degrees of joint deformity. Contrary to previous studies that investigated the QST of osteoarthritis, we included patients with not only severe joint deformity but also mild joint deformity. Our findings may explain why some patients have a high level of pain without severe radiologic deformity, and suggest that central sensitization could be a target for hip OA pain treatment.

In addition, there were no significant differences in age, sex, and BMI, but there were significant differences in pain duration, disability, and painDETECT between patients with strong/severe pain and those with mild/moderate pain. Patients with strong/severe pain tended to have prolonged pain and more severe disability. Furthermore, painful patients had high scores on painDETECT in this study. There is some evidence that patients with OA may experience neuropathic pain-like symptoms. Some patients used words such as burning and numbness to describe the pain associated with $\mathrm{OA}$; these descriptors suggest a neuropathic pain component. ${ }^{35}$ A previous study suggested that patients with high painDETECT scores, indicative of a more neuropathic element to their pain, had lower thresholds for painful mechanical punctate stimulation of an area of referred pain on the thigh. ${ }^{25}$ These patients also had greater evoked midbrain periaqueductal gray functional magnetic resonance imaging blood oxygen level-dependent signaling than patients with a low painDETECT score, suggesting that dysfunction in the central nervous system can distinguish a subset of patients with prominent central sensitization. The current study suggests that a lower PPT and facilitated TSP might be associated with neuropathic components of hip OA pain.

This study has several limitations. First, we obtained a small sample size and a single population. Further studies are required to confirm the exact influences of the pain of OA. Future studies should conduct multivariate analysis and evaluate each component of the QST and hip OA features. Second, we used only the K-L scale and mJSW to assess the joint deformity. We did not assess subchondral bone changes or synovitis. In particular, patients with strong/severe pain should be evaluated comprehensively because their pain may be complicated.

\section{Conclusion}

We assessed the central sensitization of hip OA using QST and investigated whether radiologic severity or central sensitization is more involved in hip OA pain. We found no significant differences in radiologic severity between patients with strong/severe and mild/moderate joint pain. By contrast, we found a significant difference in QST results between patients with strong/severe and mild/moderate joint pain. The results suggest that central sensitization may be involved in the joint pain of patients with hip OA who complain of severe pain despite less severe joint deformity.

\section{Ethical Approval}

Ethics approval was obtained from the institutional ethics committee of Kobe Gakuin University in Kobe, Japan. Written informed consent was obtained from all subjects before the study. This study was conducted in compliance with the Declaration of Helsinki and its later amendments. 


\section{Acknowledgments}

We would like to thank Editage for English language editing.

\section{Author Contributions}

All authors made significant contributions to this manuscript and the project in general. All authors read and approve this manuscript. We have outlined the specific contributions below:

Contributions to conception and design of the study: All authors (TH, KS, YN, YT, and TM). Drafted manuscript: TH and KS. Critically reviewed and revised the manuscript: All authors (TH, KS, YN, YT, and TM). Gave final approval of the manuscript version to be published: All authors $(\mathrm{TH}$, KS, YN, YT, and TM). Agreed to be accountable for all aspects of manuscript and trial work: All authors (TH, KS, YN, YT, and TM).

\section{Disclosure}

The authors report no conflicts of interest in this work.

\section{References}

1. Bijlsma JW, Berenbaum F, Lafeber FP. Osteoarthritis: an update with relevance for clinical practice. Lancet. 2011;377(9783):2115-2126. doi:10.1016/S0140-6736(11)60243-2

2. Dieppe PA, Lohmander LS. Pathogenesis and management of pain in osteoarthritis. Lancet. 2005;365(9463):965-973. doi:10.1016/S01406736(05)71086-2

3. Maetzel A, Li LC, Pencharz J, Tomlinson G, Bombardier C. The economic burden associated with osteoarthritis, rheumatoid arthritis, and hypertension: a comparative study. Ann Rheum Dis. 2004;63 (4):395-401.

4. Iidaka T, Muraki S, Akune T, et al. Prevalence of radiographic hip osteoarthritis and its association with hip pain in Japanese men and women: the ROAD study. Osteoarthritis Cartilage. 2016;24 (1):117-123. doi:10.1016/j.joca.2015.07.017

5. Kidd BL. Osteoarthritis and joint pain. Pain. 2006;123(1):6-9. doi:10.1016/j.pain.2006.04.009

6. Pereira D, Severo M, Santos RA, et al. Knee and hip radiographic osteoarthritis features: differences on pain, function and quality of life. Clin Rheumatol. 2016;35(6):1555-1564.

7. Hucho T, Levine JD. Signalling pathways in sensitization: toward a nociceptor cell biology. Neuron. 2007;55(3):365-376. doi:10.1016/ j.neuron.2007.07.008

8. Arendt-Nielsen L, Graven-Nielsen T. Translational musculoskeletal pain research. Best Pract Res Clin Rheumatol. 2011;25(2):209-226. doi:10.1016/j.berh.2010.01.013

9. Woolf CJ. Central sensitization: implications for the diagnosis and treatment of pain. Pain. 2011;152(3 Suppl):S2-S15.

10. Arendt-Nielsen L, Nie H, Laursen MB, et al. Sensitization in patients with painful knee osteoarthritis. Pain. 2010;149(3):573-581. doi:10.1016/j.pain.2010.04.003

11. Izumi M, Petersen KK, Laursen MB, Arendt-Nielsen L, GravenNielsen T. Facilitated temporal summation of pain correlates with clinical pain intensity after hip arthroplasty. Pain. 2017;158 (2):323-332. doi:10.1097/j.pain.0000000000000764
12. den Bandt HL, Paulis WD, Beckwée D, Ickmans K, Nijs L, Voogt L. Pain mechanisms in low back pain: a systematic review with meta-analysis of mechanical quantitative sensory testing outcomes in people with nonspecific low back pain. J Orthop Sports Phys Ther. 2019;49(10):698-715. doi:10.2519/jospt.2019.8876

13. O'Brien AT, Deitos A, Triñanes Pego Y, Fregni F, Carrillo-de-lapeña MT. Defective endogenous pain modulation in fibromyalgia: a meta-analysis of temporal summation and conditioned pain modulation paradigms. $J$ Pain. 2018;19(8):819-836. doi:10.1016/j. jpain.2018.01.010

14. Beswick AD, Wylde V, Gooberman-Hill R, Blom A, Dieppe P. What proportion of patients report long-term pain after total hip or knee replacement for osteoarthritis? A systematic review of prospective studies in unselected patients. BMJ Open. 2012;2(1):e000435. doi:10.1136/bmjopen-2011-000435

15. Suokas AK, Walsh DA, McWilliams DF, et al. Quantitative sensory testing in painful osteoarthritis: a systematic review and meta-analysis. Osteoarthritis Cartilage. 2012;20(10):1075-1085. doi:10.1016/j. joca.2012.06.009

16. Uddin Z, MacDermid JC. Quantitative sensory testing in chronic musculoskeletal pain. Pain Med. 2016;17(9):1694-1703. doi: $10.1093 / \mathrm{pm} / \mathrm{pnv} 105$

17. Kong JT, Schnyer RN, Johnson KA, Mackey S. Understanding central mechanisms of acupuncture analgesia using dynamic quantitative sensory testing: a review. Evid Based Complement Alternat Med. 2013;2013:187182. doi: $10.1155 / 2013 / 187182$

18. Wylde V, Palmer S, Learmonth ID, Dieppe P. Somatosensory abnormalities in knee OA. Rheumatology (Oxford). 2012;51 (3):535-543. doi:10.1093/rheumatology/ker343

19. Staud R, Cannon RC, Mauderli AP, Robinson ME, Price DD, Vierck CJ. Temporal summation of pain from mechanical stimulation of muscle tissue in normal controls and subjects with fibromyalgia syndrome. Pain. 2003;102(1):87-95. doi:10.1016/s0304-3959(02) 00344-5

20. Collins SL, Moore RA, McQuay HJ. The visual analogue pain intensity scale: what is moderate pain in millimetres? Pain. 1997;72 (1):95-97. doi:10.1016/S0304-3959(97)00005-5

21. Jensen MP, Mardekian J, Lakshminarayanan M, Boye ME. Validity of 24-h recall ratings of pain severity: biasing effects of "Peak" and "End" pain. Pain. 2008;137(2):422-427. doi:10.1016/j. pain.2007.10.006

22. Klassbo M, Larsson E, Mannevik E. Hip disability and osteoarthritis outcome score. An extension of the Western Ontario and McMaster Universities Osteoarthritis Index. Scand J Rheumatol. 2003;32 (1):46-51. doi:10.1080/03009740310000409

23. Satoh M, Masuhara K, Goldhahn S, Kawaguchi T. Cross-cultural adaptation and validation reliability, validity of the Japanese version of the Hip disability and Osteoarthritis Outcome Score (HOOS) in patients with hip osteoarthritis. Osteoarthritis Cartilage. 2013;21 (4):570-573. doi:10.1016/j.joca.2013.01.015

24. Freynhagen R, Baron R, Gockel U, Tolle TR. painDETECT: a new screening questionnaire to identify neuropathic components in patients with back pain. Curr Med Res Opin. 2006;22 (10):1911-1920. doi:10.1185/030079906X132488

25. Gwilym SE, Keltner JR, Warnaby CE, et al. Psychophysical and functional imaging evidence supporting the presence of central sensitization in a cohort of osteoarthritis patients. Arthritis Rheum. 2009;61(9):1226-1234. doi:10.1002/art.24837

26. Kellgren JH, Lawrence JS. Radiologic assessment of osteoarthritis. Ann Rheum Dis. 1957;16(4):494-502. doi:10.1136/ard.16.4.494

27. Croft P, Cooper C, Wickham C, Coggon D. Defining osteoarthritis of the hip for epidemiologic studies. Am J Epidemiol. 1990;132 (3):514-522. doi:10.1093/oxfordjournals.aje.a115687 
28. Nie H, Arendt-Nielsen L, Madeleine P, Graven-Nielsen T. Enhanced temporal summation of pressure pain in the trapezius muscle after delayed onset muscle soreness. Exp Brain Res. 2006;170(2):182-190. doi:10.1007/s00221-005-0196-6

29. Edwards RR, Mensing G, Cahalan C, et al. Alteration in pain modulation in women with persistent pain after lumpectomy: influence of catastrophizing. J Pain Symptom Manage. 2013;46(1):30-42. doi:10.1016/j.jpainsymman.2012.06.016

30. Cohen JL. Statistical Power Analysis for the Behavioral Sciences. Hillsday, NJ: Erlbaum; 1988.

31. Dieppe PA. Relationship between symptoms and structural change in osteoarthritis: what are the important targets for therapy? J Rheumatol. 2005;32(6):1147-1149.

32. Bosma R, Ameli Mojarad E, Leung L, Pukall C, Staud R, Stroman PW. Neural correlates of temporal summation of second pain in human brainstem and spinal cord. Hum Brain Mapp. 2015;36(12):5038-5050. doi:10.1002/hbm.22993
33. Hoheisel U, Mense S. Response behaviour of cat dorsal horn neurones receiving input from skeletal muscle and other deep somatic tissues. J Physiol. 1990;426:265-280. doi:10.1113/jphysiol.1990. sp018137

34. Hoheisel U, Mense S, Simons DG, Yu XM. Appearance of new receptive fields in rat dorsal horn neurons following noxious stimulation of skeletal muscle: a model for referral of muscle pain? Neurosci Lett. 1993;153(1):9-12. doi:10.1016/0304-3940(93)90064-R

35. Blikman T, Rienstra W, Raay JJAM, et al. Neuropathic-like symptoms and the association with joint-specific function and quality of life in patients with hip and knee osteoarthritis. PLoS One. 2014;13 (6):e0199165. doi:10.1371/journal.pone.0199165

\section{Publish your work in this journal}

The Journal of Pain Research is an international, peer reviewed, open access, online journal that welcomes laboratory and clinical findings in the fields of pain research and the prevention and management of pain. Original research, reviews, symposium reports, hypothesis formation and commentaries are all considered for publication. The manuscript management system is completely online and includes a very quick and fair peer-review system, which is all easy to use. Visit http:// www.dovepress.com/testimonials.php to read real quotes from published authors. 